\title{
Bicycle Use for Transport in an Australian and a Belgian City: Associations with Built-Environment Attributes
}

\author{
Neville Owen, Ilse De Bourdeaudhuij, Takemi Sugiyama, Eva Leslie, \\ Ester Cerin, Delfien Van Dyck, and Adrian Bauman
}

\begin{abstract}
The walkability attributes of neighborhood environments (residential density, land use mixture, and connectedness of streets) have been found to be associated with higher rates of walking. However, relatively less is known about the associations of walkability attributes with bicycle use for transport. We examined the relationships between adults' bicycle use for transport and measures of neighborhood walkability in two settings: an Australian city (Adelaide) with low rates of bicycle use and a Belgian city (Ghent) with high rates of bicycle use. A total of 2,159 and 382 participants were recruited in Adelaide and Ghent, respectively. A walkability index was derived from objectively measured data in Adelaide, while a similar index was derived from perceived measures in Ghent. Logistic regression models were employed to examine associations of bicycle use with different levels of walkability. There were higher rates of bicycle ownership for Ghent compared to Adelaide participants (96\% versus 61\%), and there was a higher prevalence of bicycle use for transport for Ghent compared to Adelaide participants (50\% vs. 14\%). Despite the large differences in bicycle ownership and use, living in a high-walkable neighborhood was associated with significantly higher odds of bicycle use for transport in both cities, after adjusting for relevant confounding factors. Built-environment innovations that are increasingly being advocated by health authorities and transport planners, primarily to promote higher rates of walking for transport, should also impact positively on bicycle use.
\end{abstract}

KEYWORDS Physical activity, Cycling, Neighborhood walkability

\section{INTRODUCTION}

Participation in physical activity has many significant health-protective benefits. ${ }^{1,2}$ Public health guidelines identify the importance of $30 \mathrm{~min}$ or more of moderateintensity activities on most days of week, typically emphasizing regular walking, either for the purpose of recreation, exercise, or transport. ${ }^{2-4}$ Bicycle use for transport is a form of physical activity that can make significant contributions to

Owen and Sugiyama are with the Cancer Prevention Research Centre, School of Population Health, The University of Queensland, Herston, QLD, Australia; De Bourdeaudhuij and Van Dyck are with the Department of Movement and Sport Sciences, Ghent University, Ghent, Belgium; Leslie is with the School of Psychology, Deakin University, Geelong, Australia; Cerin is with the Institute of Human Performance, The University of Hong Kong, Hong Kong; Bauman is with the Centre for Physical Activity and Health, School of Public Health, The University of Sydney, Sydney, Australia.

Correspondence: Neville Owen, Cancer Prevention Research Centre, School of Population Health, The University of Queensland, Herston Road, Herston, QLD 4006, Australia. (E-mail: n.owen@sph.uq.edu.au) 
overall health-enhancing physical activity ${ }^{5}$ and can have an independent influence on health outcomes: for example, a large prospective study carried out in Copenhagen, Denmark $^{6}$ showed a $30 \%$ lower odds of premature death at follow-up among adults who bicycled regularly to work, after adjustment for a number of potential confounding factors. Compared to European cities, rates of bicycle use among urban adults are much lower in Australia. ${ }^{7}$ Nevertheless, a recent study in Australia showed an association of bicycle use for transportation with a significantly lower risk of obesity. ${ }^{8}$ Additionally, adverse health effects of prolonged driving time have been identified in recent studies, ${ }^{9,10}$ which further strengthens the case for active transport (including bicycle use) as an important element of chronic disease prevention strategies. In order to develop the most appropriate initiatives to increase bicycle use, modifiable factors associated with participation in this behavior need to be identified.

The factors found to be most consistently associated with adults' overall physical activity are demographic attributes: men and younger people are typically more likely to be physically active. ${ }^{11}$ Adults of higher socioeconomic status (SES) are more active in their leisure time, but there is more walking for transportation among those of lower SES. ${ }^{12}$ Consistent with ecological models of health behavior, ${ }^{13-17}$ there is also the need to identify influences on physical activity that should be amenable to environmental and policy innovations. These ecological models focus on particular behaviors (for example, walking for transport as distinct from walking for recreation exercise and bicycle commuting as distinct from recreational cycling), which may be influenced by particular environmental attributes. Built-environment attributes that have been found to be associated consistently with active transportation choices are proximity to destinations and the connectivity of street networks. ${ }^{18-21}$ Proximity relates to more mixed land use that creates shorter distances between residences and destinations for walking and cycling, such as stores, services, public transport stops, or work places. Connectivity involves street configuration that also affects distances to destinations; grid patterns of interconnecting streets tend to provide more direct and shorter routes to destinations, compared to cul-de-sac-type street layouts. The concept of "walkability," which incorporates these built-environment attributes, has been developed to assess the extent to which neighborhood environments facilitate walking. ${ }^{20,22,23}$ Both objective and perceived environmental attributes have been shown to be associated with adults' participation in physical activity, after adjusting for a wide range of personal, social, and demographic attributes. ${ }^{24}$

Although substantial evidence indicates the association of neighborhood walkability with residents' walking, ${ }^{20,22,23}$ relatively less is known about the associations of walkability attributes with adults' bicycle use for transport. It can be hypothesized that higher walkability is also conducive to greater bicycle use. We examined the associations of built-environment attributes with bicycle use in contextually contrasting settings: an Australian city where rates of bicycle use are low and a European city where rates of bicycle use are high.

\section{METHODS}

\section{Survey Methods}

Two cross-sectional studies were conducted in Adelaide, Australia and in Ghent, Belgium. The original purpose of these studies was to identify psychosocial and environmental correlates of adults' physical activity. 
Adelaide Sample Participants were randomly recruited from 32 communities in the city of Adelaide (population is approximately 1.1 million) between 2003 and 2004. These communities were chosen from high- and low-walkable communities, which were the top and bottom quartiles of communities ranked by "walkability" score (a composite environmental index derived from objective measures of residential density, street connectivity, land use mix, and net retail area ratio), respectively. Using mailed survey, data were collected from 2,159 adults aged between 20 and 65 years old. The overall response rate (as a ratio of eligible participants to identified households in the chosen communities) was $11.5 \%$. Accounts of the recruitment methods are reported elsewhere. ${ }^{25}$

Ghent Sample In 2003, a convenience sample of adults (18-65 years old) was recruited through worksites, libraries, and community organizations in the city of Ghent, Belgium. Ghent is a city in the northern part of Belgium with 224,000 inhabitants. The sample consisted of 382 participants. A more detailed description has been previously reported. ${ }^{26}$

\section{Sociodemographic Attributes}

In both studies, survey respondents provided information on their age, gender, educational attainment, and whether they were working or not.

\section{Outcome Measure (Bicycle Use for Transport)}

In both the Adelaide and the Ghent studies, bicycle use for transport was measured using the relevant item from the International Physical Activity QuestionnaireLong Form (IPAQ). ${ }^{27}$ This instrument is designed for use by adults, and the long form assesses physical activity in specific domains including transport-related and leisure time. ${ }^{27}$ The reliability and validity of the overall IPAQ instrument has been tested across 12 countries. ${ }^{28}$ Bicycle use for transport is assessed using the following item: "During the last seven days, on how many days a week did you bicycle for at least 10 minutes at a time to go from place to place?" In both cities, participants were classified into those who reported using a bicycle at least once a week for this purpose (current bicycle users) and those who did not.

\section{Environmental Attributes (Walkability)}

Adelaide Study Walkability was assessed at the level of Census Collection Districts (CDs) via Geographic Information System databases (ArcGIS) using four environmental attributes found to be related to walking: dwelling density, street connectivity, land use mix, and net retail area ratio. ${ }^{18,19}$ Street centerline data, land use, zoning data, shopping center location data, and population census data were spatially integrated within a GIS to create a composite measure of "walkability index" for each CD. ${ }^{23}$ Each of the four environmental attributes was classified into deciles to provide a score from 1 to 10, with 1 indicating low walkability and 10 indicating high walkability. The walkability index was derived by summing the decile scores for the four relevant walkability attribute measures, resulting in a possible score range of 4 to 40 . Then, walkability of "community," which consists of several (three to nine) contiguous CDs, was calculated as the mean of CD walkability scores. The resulting walkability index was further recoded into quartiles, with the first quartile used to identify low-walkable CDs and the fourth quartile identifying high-walkable CDs. The 32 communities that we examined in our study were classified as high or low walkable on this basis. 
Similar walkability indexes have been examined in relation to physical activity variables in studies conducted in Atlanta, USA. ${ }^{20}$ In addition to walkability, area-level SES was also determined for each CD using median household weekly income.

Ghent Study Perceptions of the physical environment were assessed with a 40-item questionnaire, which was a modified Dutch version of the Neighborhood Environment Walkability Scale instrument, developed in the USA to assess perceived neighborhood environmental attributes associated with physical activity. ${ }^{29}$ With this questionnaire, individual perceptions of physical environmental features in the neighborhood were assessed. "Neighborhood" was defined as the local geographical area, which typically would be within a 10 - to 15 -min walk around the participant's home. Neighborhood environmental variables assessed included residential density, land use mix (distance to facilities), and connectivity, based on which a walkability index was computed. The sum of these scores was recoded into quartiles, with the lowest quartile representing the lowest walkability and the highest quartile the highest walkability. A separate study showed good reliability and validity of this questionnaire in Belgium. ${ }^{26}$

\section{Statistical Analyses}

Percentages and mean scores were used to report the characteristics of both samples and the proportions of adults using bicycles for transport. Logistic regression models were used to examine associations of current bicycle use with walkability. Analyses adjusted for age, gender, education, working status, and for Adelaide only, area-level SES. Since cluster sampling was used in Adelaide, individuals in the same community shared the same objectively measured environmental attributes. Thus, the Adelaide dataset had a two-level structure: individuals (level 1) nested within 32 communities (level 2). Multilevel logistic modeling was used to analyze the Adelaide data. The model was calibrated through the binomial logit link model using the second-order penalized quasi-likelihood estimation procedure. ${ }^{30}$ The logits were transformed to odds ratios (ORs). The Ghent sample did not have a multilevel structure. Analyses were conducted using SPSS version 13.0 for general analysis and MLwiN version 2.02 for multilevel analysis.

\section{RESULTS}

Table 1 shows the characteristics of those in our study samples. After excluding missing values, a sample of 1,940 adults in Adelaide and 372 in Ghent were analyzed. Compared to the 2001 census data, ${ }^{31}$ those in the Adelaide sample were more likely to be older, female, and working. In the Ghent sample, the sample was more educated compared to the 2004 census data. ${ }^{32}$ Bicycle ownership was reported by $61 \%$ of the respondents in Adelaide and by $96 \%$ in Ghent. No differences in bicycle ownership were found between men and women. Overall, $14 \%$ of the Adelaide sample and $50 \%$ of the Ghent sample reported that they used bicycle at least once a week for transport. In Ghent, more than half of the men reported themselves to be regular bicycle users, compared to a somewhat smaller proportion of the women (men, 57\%; women, $43 \%, p<0.05$ in $\chi^{2}$ analysis). Much lower rates of bicycle use were reported in Adelaide (men, 21\%; women, 9\%, $p<0.001$ in $\chi^{2}$ analysis).

In Adelaide, bicycle use was more common for younger age groups of men and women (see Table 2). By contrast, in Ghent, no significant differences in bicycle use were found between age groups. In Ghent, no differences in bicycle use were found 
TABLE 1 Characteristics of the Adelaide and Ghent samples

\begin{tabular}{|c|c|c|c|c|}
\hline & \multicolumn{2}{|c|}{ Adelaide } & \multicolumn{2}{|c|}{ Ghent } \\
\hline & Men $(n=730)$ & Women $(n=1,210)$ & Men $(n=183)$ & Women $(n=189)$ \\
\hline $\begin{array}{l}\text { Mean age, years } \pm S D \\
\text { Education }(\%)\end{array}$ & $48.1 \pm 11.5$ & $43.7 \pm 11.9$ & $46.9 \pm 15.7$ & $44.8 \pm 12.1$ \\
\hline $\begin{array}{l}\text { Tertiary education } \\
\text { Working or not (\%) }\end{array}$ & 46 & 48 & 55 & 48 \\
\hline Working & 70 & 64 & 74 & 77 \\
\hline Bicycle owners (\%) & 62 & 61 & 98 & 95 \\
\hline Current bicycle users (\%) & 21 & 9 & 57 & 43 \\
\hline
\end{tabular}

for education, but differences were found between women working and not working. Working women were less frequent bicycle users than nonworking women. In Adelaide, bicycle use differed between higher and lower educational attainment and between workers and nonworkers significantly for men, but not for women. More cycling was reported by higher-educated men and by working men.

The logistic regression models (Table 3) supported our key hypothesis. After adjusting for potential confounders, higher neighborhood walkability was associated with a higher likelihood of bicycle use for transport, in both the Adelaide and Ghent samples, despite the large differences in rates of bicycle ownership and bicycle use. In Adelaide, compared to living in a lower-walkable community, those living in a higher-walkable community had $82 \%$ higher odds of regular bicycle use for transport. In Ghent, those living in the higher and highest walkability neighborhoods had approximately a 2.5 times higher likelihood of using bicycle for transport, compared to those living in the lowest walkability neighborhoods ( $p$ for trend $=0.004$ ). Residents of areas in the highest walkability quartile had more than 2.5 times higher

TABLE 2 Proportions of adults currently using a bicycle for transport at least once a week, by individual-level attributes

\begin{tabular}{|c|c|c|c|c|c|c|}
\hline & \multicolumn{3}{|c|}{ Adelaide } & \multicolumn{3}{|c|}{ Ghent } \\
\hline & Men & Women & Total & Men & Women & Total \\
\hline \multicolumn{7}{|l|}{ Age in years } \\
\hline 20-36 (\%) & 27 & 12 & 16 & 62 & 43 & 53 \\
\hline $37-46(\%)$ & 23 & 12 & 16 & 51 & 34 & 41 \\
\hline $47-55(\%)$ & 23 & 6 & 13 & 54 & 49 & 51 \\
\hline $56-65(\%)$ & 14 & 5 & 10 & 57 & 52 & 56 \\
\hline$p$ value $^{\mathrm{a}}$ & $<0.05$ & $<0.01$ & $<0.01$ & 0.76 & 0.33 & 0.23 \\
\hline \multicolumn{7}{|c|}{ Tertiary education } \\
\hline No (\%) & 18 & 8 & 12 & 60 & 41 & 50 \\
\hline Yes (\%) & 25 & 11 & 16 & 54 & 45 & 50 \\
\hline$p$ value $^{\mathrm{a}}$ & $<0.05$ & 0.09 & $<0.05$ & 0.44 & 0.59 & 1.00 \\
\hline \multicolumn{7}{|l|}{ Working } \\
\hline No (\%) & 15 & 8 & 10 & 65 & 57 & 61 \\
\hline Yes (\%) & 23 & 10 & 16 & 54 & 39 & 46 \\
\hline$p$ value $\mathrm{e}^{\mathrm{a}}$ & $<0.05$ & 0.12 & $<0.01$ & 0.19 & $<0.05$ & $<0.05$ \\
\hline
\end{tabular}

${ }^{a} X^{2}$ analysis 
TABLE 3 Logistic regression models predicting current bicycle use for transport at least once a week (ORs and 95\% confidence intervals)

\begin{tabular}{lll}
\hline & \multicolumn{1}{c}{ Adelaide } & \multicolumn{1}{c}{ Ghent } \\
\hline Age in years & & 1.00 \\
$20-36$ & 1.00 & $0.62(0.36-1.09)$ \\
$37-46$ & $0.86(0.60-1.22)$ & $0.93(0.54-1.62)$ \\
$47-55$ & $0.66(0.45-0.95)^{*}$ & $1.13(0.62-2.06)$ \\
$56-65$ & $0.43(0.28-0.66)^{* * *}$ & \\
Gender & 1.00 & 1.00 \\
$\quad$ Men & $0.34(0.26-0.45)^{* * *}$ & $0.58(0.39-0.88)^{*}$ \\
$\quad$ Women & 1.00 & 1.00 \\
Tertiary education & $1.04(0.77-1.41)$ & $1.00(0.66-1.51)$ \\
$\quad$ No & 1.00 & 1.00 \\
$\quad$ Yes & $1.25(0.91-1.73)$ & $0.54(0.33-0.88)^{*}$ \\
Working & 1.00 & - \\
$\quad$ No & $1.14(0.77-1.69)$ & - \\
$\quad$ Yes & & 1.00 \\
Area-level SES & 1.00 & $1.52(0.86-2.70)$ \\
Lower & - & $2.42(1.34-4.38)^{* * *}$ \\
Higher & - & $2.62(1.45-4.72)^{* * *}$ \\
Walkability & $1.82(1.24-2.66)^{* *}$ & \\
$\quad$ Lowest & High (Ghent only) &
\end{tabular}

Analyses adjusted for age, gender, education, working status, and for Adelaide only, area-level SES ${ }^{*} p<0.05 ;{ }^{* *} p<0.01 ;{ }^{* *} p<0.001$

likelihood of using a bicycle for transport, compared to those living in the lowest walkability quartile. Table 3 also shows statistically significant associations for gender in both samples, with women having a lower likelihood of bicycle use compared to men. In Adelaide, a clear age gradient was found, with less bicycling with increasing age; this was not the case for Ghent. In Ghent, those who are working also reported less bicycle use, compared to those who were not working.

\section{DISCUSSION}

We examined whether living in more walkable neighborhoods was associated with adults using a bicycle for transport in a city with low bicycle use rates (Adelaide, Australia) and in a city with higher bicycle use rates (Ghent, Belgium). In both samples, after adjusting for age, gender, educational attainment, and working status, we found that those living in higher-walkable communities had significantly higher odds of bicycle use for transport, despite the large differences between countries in cycling ownership and use. This relationship was consistent, using different ways in assessing the walkability of the local neighborhoods for both cities. In Adelaide, a walkability index was derived from objective data, while in Ghent, a similar index was derived from perceived measures. Our findings on bicycle use are consistent with those of other studies on the relationships of community environment attributes with adults' walking for transport. ${ }^{14,16,33}$ 
Although studies have shown that the availability of bicycle lanes is related to cycling, ${ }^{34,35}$ only a few studies have investigated cycling for transport and its relationship with walkability attributes. For instance, a study in the USA found that street connectivity was associated with greater bicycle use for utilitarian purposes (unadjusted analysis). ${ }^{36}$ An Austrian study has also shown a significant relationship between bike lane connectivity and cycling for transportation. ${ }^{37}$ Our findings extend these studies by demonstrating associations of neighborhood walkability with bicycle use within the context of Australian and Belgian urban environments where the prevalence of bicycle use varies considerably.

As is the case for other health behaviors, ${ }^{13,15}$ bicycle use will be determined in part by individual-level factors such as age, gender, and work status, as our findings demonstrate. These personal attributes are likely to operate in conjunction with broader social, cultural, and environmental factors ${ }^{13,15}$ to determine the likelihood of bicycle use. Thus, in efforts to promote higher levels of bicycle use, there is the need to work simultaneously on advocacy for changing environments to make bicycle use an easier choice and to encourage public campaigns, social marketing initiatives, and other approaches that can inform and motivate individuals to take up bicycling as an active transportation option. ${ }^{38}$

There are some limitations in our comparative study findings. Due to the crosssectional design, causal relationships between walkability and bicycle use cannot be inferred from this study. Although bicycle use is unlikely to have a direct impact on walkability, those who cycle to get to places may have chosen to live in highwalkable neighborhoods. Different measures were used in Adelaide and Ghent to obtain walkability. Although they aimed to capture the same aspects of neighborhood environments (e.g., density, connectivity, and land use mixture), the Adelaide study used objective measures and the Ghent study used perception. The size of the area within which walkability was determined also differed between these studies. It has been reported that residents' perception agrees to some extent with objectively measured walkability. ${ }^{39}$ However, the different methods used to identify walkability may have had an impact on the results of the study. The low response rate in the Adelaide study is another limitation of the study. Since the request to participate in the study was sent to households, which included ineligible households (those without persons between 20 and 65 years old), the actual response rate could have been higher than $11.5 \%$. However, it could have introduced a selection bias. Ideally, future comparative studies should use more similar sample sizes than those available to us, using the data from the two quite different studies on which our analyses are based. Our measure of bicycle use does not allow us to consider directly how these findings relate to health-enhancing physical activity. Participants were classified as those who reported using a bicycle at least once a week for transport (current bicycle users) and those who did not. It must be acknowledged that this is a modest criterion in the perspective of recommendations on overall health-related physical activity (30 min/day on most days of the week). Finally, these findings may not be generalizable to the broader population of Australian or Belgian cities as both samples were different from the city population in a number of sociodemographic characteristics.

In both the Adelaide and Ghent studies, several potential psychosocial correlates of bicycle use were not included in our measures. As a consequence, we cannot identify all the possible influences of interactions between unmeasured psychosocial and environmental factors, and in particular, the unique cultural factors that vary between countries. The results of a recent study in Belgium ${ }^{40}$ suggest that individual 
and social factors are more strongly predictive in distinguishing between cyclists and noncyclists than are measures of built-environment attributes. However, the findings reported here showed that the more "objective" walkability index used does show an independent relationship with cycling. Another study has suggested that certain psychosocial factors (social support and perceptions of safety), as well as environmental attributes, are important in explaining adults' transport-related bicycle use. ${ }^{37}$ Further studies of the interactions between environmental and psychosocial determinants of cycling are warranted.

There is the potential to promote healthier, physically active lifestyles among large numbers of adults through more active transport choices including walking and cycling. Enhancing the neighborhood walkability attributes examined in this study is a potentially effective approach in this regard as it is likely to increase not only walking but also bicycle use for transportation.

\section{ACKNOWLEDGEMENTS}

For the Adelaide study, we thank the South Australian Government Department for Transport and Urban Planning for providing access to the relevant GIS data. The National Health and Medical Research Council (NHMRC) Project Grant \#213114 and NHMRC Program Grant \#301200 supported the Physical Activity in Localities and Community Environments (PLACE) study. Sugiyama is supported by a Capacity Building Grant in Population Health (\#252799) from the NHMRC. Leslie is supported by an NHMRC Public Health Fellowship (\#301261). For the Ghent study, we thank the Ghent University for the grant support for Van Dyck. We also thank Professors Graeme Hugo, James F. Sallis, Lawrence Frank, and Neil Coffee for their conceptual, methodological, and practical contributions.

\section{REFERENCES}

1. Bauman A, Bellew B, Vita P, Brown W, Owen N. Getting Australia Active: Best Practice for the Promotion of Physical Activity. Melbourne: National Public Health Partnership; 2002.

2. United States Department of Health and Human Services. Physical Activity and Health: A Report of the Surgeon General. Atlanta: Public Health Service, Centers for Disease Control and Prevention, National Centre for Chronic Disease Prevention and Health Promotion; 1996.

3. Bauman AE. Updating the evidence that physical activity is good for health: an epidemiological review 2000-2003. J Sci Med Sport. 2004; 7(1 Suppl): 6-19.

4. Sallis JF, Owen N. Physical Activity and Behavioral Medicine. Thousand Oaks: Sage; 1999.

5. Oja P, Vuori I, Paronen O. Daily walking and cycling to work: their utility as healthenhancing physical activity. Patient Educ Couns. 1998; 33(1 Suppl): S87-S94.

6. Andersen LB, Schnohr P, Schroll M, Hein HO. All-cause mortality associated with physical activity during leisure time, work, sports and cycling to work. Arch Intern Med. 2000; 160(11): 1621-1628.

7. Newman PWG, Kenworthy JR. Transport and urban form in 32 of the worlds principal cities. Transport Rev. 1991; 11(3): 249-272.

8. Wen LM, Rissel C. Inverse associations between cycling to work, public transport, and overweight and obesity: findings from a population based study in Australia. Prev Med. 2008; 46(1): 29-32.

9. Lopez-Zetina J, Lee H, Friis R. The link between obesity and the built environment: evidence from an ecological analysis of obesity and vehicle miles of travel in California. Health Place. 2006; 12(4): 656-664. 
10. Wen LM, Orr N, Millett C, Rissel C. Driving to work and overweight and obesity: findings from the 2003 New South Wales Health Survey, Australia. Int J Obes. 2006; 30 (5): 782-786.

11. Trost SG, Owen N, Bauman AE, Sallis JF, Brown W. Correlates of adults participation in physical activity: review and update. Med Sci Sports Exerc. 2002; 34(12): 1996-2001.

12. Cole R, Leslie E, Bauman A, Donald M, Owen N. Socio-demographic variations in walking for transport and for recreation or exercise among adult Australians. J Phys Activ Health. 2006; 3(2): 164-178.

13. Giles-Corti B, Timperio A, Bull F, Pikora T. Understanding physical activity environmental correlates: increased specificity for ecological models. Exerc Sport Sci Rev. 2005; 33(4): 175-181.

14. Saelens BE, Sallis JF, Frank LD. Environmental correlates of walking and cycling: findings from the transportation, urban design, and planning literatures. Ann Behav Med. 2003; 25(2): 80-91.

15. Sallis JF, Owen N. Ecological models of health behavior. In: Glanz K, Lewis FM, Rimer BK, eds. Health Behavior and Health Education: Theory, Research and Practice. 3rd ed. San Francisco: Jossey-Bass; 2002: 462-484.

16. Owen N, Humpel N, Leslie E, Bauman A, Sallis JF. Understanding environmental influences on walking: review and research agenda. Am J Prev Med. 2004; 27(1): 67-76.

17. Owen N, Leslie E, Salmon J, Fotheringham MJ. Environmental determinants of physical activity and sedentary behavior. Exerc Sport Sci Rev. 2000; 28(4): 153-158.

18. Frank LD, Engelke PO, Schmid TL. Health and Community Design: The Impact of the Built Environment on Physical Activity. Washington: Island; 2003.

19. Leslie E, Coffee N, Frank L, Owen N, Bauman A, Hugo G. Walkability of local communities: using geographic information systems to objectively assess relevant environmental attributes. Health Place. 2007; 13(1): 111-122.

20. Frank LD, Schmid TL, Sallis JF, Chapman J, Saelens BE. Linking objectively measured physical activity with objectively measured urban form: findings from SMARTRAQ. Am J Prev Med. 2005; 28(2 Suppl 2): 117-125.

21. Ewing R, Cervero R. Travel and the built environment: a synthesis. Transport Res Rec. 2001; 1780: 87-114.

22. Doyle S, Kelly-Schwartz A, Schlossberg M, Stockard J. Active community environments: the relationship of walkable and safe communities to individual health. J Am Plann Assoc. 2006; 72(1): 19-31.

23. Owen N, Cerin E, Leslie E, Bauman A, Sallis JF. Neighbourhood walkability and the walking behavior of Australian adults. Am J Prev Med. 2007; 33: 387-395.

24. McGinn AP, Evenson KR, Herring AH, Huston SL, Rodriguez DA. Exploring associations between physical activity and perceived and objective measures of the built environment. J Urban Health. 2007; 84(2): 162-184.

25. du Toit L, Cerin E, Leslie E. An Account of Spatially based Survey Methods and Recruitment Outcomes of the Physical Activity in Localities and Community Environments (PLACE) Study. Brisbane: Cancer Prevention Research Centre, School of Population Health, The University of Queensland; 2005. Available at: http://www.uq.edu.au/cprc/ docs/Place_report_2005_Final.pdf. Accessed on November 28, 2008.

26. De Bourdeaudhuij I, Teixeira PJ, Cardon G, Deforche B. Environmental and psychosocial correlates of physical activity in Portuguese and Belgian adults. Public Health Nutr. 2005; 8: 886-895.

27. International Physical Activity Questionnaire. Guidelines for data processing and analysis of International Physical Activity Questionnaire (IPAQ). 2005. Available at: http://www.ipaq.ki.se/ dloads/IPAQ\%20LS\%20Scoring\%20Protocols_Nov05.pdf. Accessed on December 8, 2008.

28. Craig CL, Marshall AL, Sjöström M, et al. International physical activity questionnaire: 12-country reliability and validity. Med Sci Sports Exerc. 2003; 35(8): 1381-1395.

29. Saelens BE, Sallis JF, Black JB, Chen D. Neighborhood-based differences in physical activity: an environment scale evaluation. Am J Public Health. 2003; 93(9): 1552-1558. 
30. Goldstein H. Multilevel Statistical Models. London: Arnold; 2003.

31. Australian Bureau of Statistics. Census of Population and Housing: CDATA 2001, CD-ROM, Final Release. Canberra: ABS; 2001.

32. National Institute of Statistics. Economie-Algemene Directie Statistiek. Available at: http://www.statbel.fgov.be. Accessed on July 4, 2008.

33. McCormack GR, Giles-Corti B, Bulsara M. The relationship between destination proximity, destination mix and physical activity behaviors. Prev Med. 2008; 46(1): 33-40.

34. Krizek KJ, Johnson PJ. Proximity to trails and retail: effects on urban cycling and walking. J Am Plann Assoc. 2006; 72(1): 33-42.

35. Garrard J, Rose G, Lo SK. Promoting transportation cycling for women: the role of bicycle infrastructure. Prev Med. 2008; 46(1): 55-59.

36. Dill J, Voros K. Factors affecting bicycling demand: initial survey findings from the Portland, Oregon, region. Transport Res Rec. 2007; 2031(1): 9-17.

37. Titze S, Stronegger WJ, Janschitz S, Oja P. Environmental, social, and personal correlates of cycling for transportation in a student population. J Phys Activ Health. 2007; 4(1): 66-79.

38. Broeg W, Erl E, Otto K, Sammer G. Promotion and planning for bicycle transportation: an international overview. Transport Res Rec. 1984; 959: 73-79.

39. Leslie E, Saelens B, Frank L, et al. Residents' perceptions of walkability attributes in objectively different neighbourhoods: a pilot study. Health Place. 2005; 11: 227-236.

40. De Geus B, De Bourdeaudhuij I, Jannes C, Meeusen R. Psychosocial and environmental factors associated with cycling for transport among a working population. Health Educ Res. 2008; 23(4): 697-708. 